\title{
Have the "mega-journals" reached the limits to growth?
}

Bo-Christer Björk

A "mega-journal" is a new type of scientific journal that publishes freely accessible articles, which have been peer reviewed for scientific trustworthiness, but leaves it to the readers to decide which articles are of interest and importance to them. In the wake of the phenomenal success of PLOS ONE, several other publishers have recently started megajournals. This article presents the evolution of mega-journals since 2010 in terms of article publication rates. The fastest growth seems to have ebbed out at around 35,000 annual articles for the 14 journals combined. Acceptance rates are in the range $50-70 \%$ and speed of publication 3-5 months. Common features in mega-journals are alternative impact metrics, easy reusability of figures and data, post-publication discussions and portable reviews from other journals. 


\section{Have the "Mega-Journals" Reached the Limits to Growth?

\section{Abstract}

7 A "mega-journal" is a new type of scientific journal that publishes freely accessible articles, which 8 have been peer reviewed for scientific trustworthiness, but leaves it to the readers to decide

9 which articles are of interest and importance to them. In the wake of the phenomenal success of

10 PLOS ONE, several other publishers have recently started mega-journals. This article presents

11 the evolution of mega-journals since 2010 in terms of article publication rates. The fastest

12 growth seems to have ebbed out at around 35,000 annual articles for the 14 journals combined.

13 Acceptance rates are in the range 50-70\% and speed of publication 3-5 months. Common

14 features in mega-journals are alternative impact metrics, easy reusability of figures and data, 15 post-publication discussions and portable reviews from other journals.

Bo-Christer Björk,

20 Hanken School of Economics,

21 Department of Management and Organisation,

22 Helsinki, Finland

23 Bo-Christer.Bjork@hanken.fi 


\section{Introduction}

27 The scientific scholarly journal emerged in the $17^{\text {th }}$ century and evolved as an institution into its 28 current form in the mid $20^{\text {th }}$ century. A traditional scientific journal appears with a number of regular issues, the format of the articles is more or less standardized, depending on the subject field, and the way articles are accepted and edited follows peer review routines which most 
Journal of the American Medical Society). And some of the world-leading scientific journals are extremely broad (Nature). After the second world war in particular the leading commercial publishers started creating lot of "niche" journals picking up new emerging research themes (Electronic Commerce Research and Applications). In the social sciences and humanities journals have sometimes been established for extremely narrow purposes (Nordic Wittgenstein Review).

The early successful electronic only $\mathrm{OA}$ journals where usually of the narrow variety, often focusing on phenomena relating to the Internet (Journal of Medical Internet Research). When new professional OA publishers emerged, they at first strived to launch journals using the same peer review mechanisms and criteria as traditional ones (PLOS Biology). In 2006, the OA publisher Public Library of Science launched a new type of journal, which later, due to its success, was been termed a "mega-journal" (the term has also been spelled megajournal or mega journal, in this article mega-journal will be used). PLOS ONE incorporates into its business model a number of features which all have been used or at least experimented with before but never in this unique combination. These features have been discussed by a number of authors (Frank, 2012; Binfield 2014). The central criterion is a new type of peer review, which only checks that the research methods are scientifically trustworthy, but does no evaluation of the perceived scientific impact or contribution. Provided that the quality of submissions overall is reasonable this leads to lower rejection rate than usual. Together with the broad scope this opens up the opportunity for very big publication volumes.

Inspired by the phenomenal growth of PLOS ONE several reputable publishers have in the past 34 years started similar journals, all apparently aiming at annual publication volumes of hundreds if not thousands of articles. Since this is a very recent phenomenon hardly any research has been done about it. The aims of this study were to:

- Identify currently existing meta-journals

- Estimate the number of articles published longitudinally since 2010.

- Collect data about the publication charges

- Collect data about the acceptance rates

- Collect information about the lead times from submission to publication Study other aspects of interest

\section{Methods}

It is unclear to this author who first coined the phrase mega-journal, and hence first discussed what criteria such a journal should fulfill. The Wikipedia article on the topic starts out by saying that "A mega journal is a peer-reviewed academic open access journal designed to be much larger than a traditional journal by exerting low selectivity among accepted articles" (Wikipedia, $2015)^{2}$. This sentence conveys the rationale for the choice of the term mega, indicating a business plan to publish clearly more articles than scholarly journals usually do. The articles by Binfield (2014) and Frank (2012) also include explicit lists of criteria. These sources have been taken into

${ }^{2}$ The author wishes to emphazise that he hasn't made any contribution to the Wikipedia article 
110 consideration in synthesizing the definition below, used in the study at hand. The three columns 111 indicate which criteria have been mentioned in the three sources above.

112

113

114

115

116

117

118

119

120

121

122

123

124

125

126

127

128

129

130

131

132

133

134

135

136

137

138

139

140

141

142

143

144

145

146

147

148

149

150

151

152

153

154

\section{Table 1. Criteria for a mega-journal used in this study}

Binfield and Frank do not use exactly the same terms as above, both for instance talk indirectly about the aim for a big publishing volume in discussing automated workflows, marginal cost for each article below the APC, etc.

The criteria have been grouped into two levels: primary and secondary. In order to qualify as a mega-journal a journal has to fulfill all the primary criteria. A mega-journal should also fulfill most of the secondary criteria, although they are not mandatory. The APC is in this study considered moderate if it's 1500 USD or below. This is around the average article processing charge currently paid by academics in Western European Countries (Björk \& Solomon, 2014) and slightly above the price charge by mega-journal market leader PLOS ONE. The publisher prestige criterion is important in an indirect way since it is a very important factor in attracting manuscripts early on and in assuring rapid inclusion in the Web of Science. The impact factor then further accelerates the growth of the journal. This criterion thus helps in identifying strong mega-journal candidates before they have reached high publication volumes and even in the announcement stage. Academic editors means that academics, not professional employed staff act as editors of the individual manuscripts.

Many mega-journals contain value-adding features, which for the time being mostly are lacking in traditional journals (even in the electronic versions). The reusability of graphics and data is facilitated by the use of Creative Commons licenses, which allows readers to reuse materials without explicitly asking for permission, and technically by having options for downloading images in high resolution for reuse in say presentation slides. Public Library of Science has been a pioneer for what is often termed "Altmetrics" (Priem et al., 2010). This means that the article contains constantly updated data about citations, bookmarks and tweets. Authors (as well as readers) can get also detailed breakdowns of how the article has been downloaded. The possibility for readers to comment on articles provides a sort of open peer review. Also authors can use such facilities for responding.

An interesting but somewhat controversial practice that several mega-journals are adopting is reuse of reviews from journals, which have rejected the manuscript in question. The terms cascading and portable reviews have been used to describe this (Davis, 2010). Usually the originating journals are more selective journals of the same publisher but in some cases even cross-publisher co-operation has been announced (Clarke, 2013).

A recent systematic study of publishing delays in different scientific fields (Björk \& Solomon 2013) found average publishing delays of between 9 and 18 months depending of the field of science. In biomedicine, the area of a majority of mega-journal articles, the average was 9,5 months). For OA journals the average was 6 months. In the context of this study rapid publication was defined as less than half a year from the original submission. 
155 The identification of mega-journals that fulfill the primary criteria and most of the secondary ones, was based on the author's previous knowledge of the OA market, on web searches and on tracking citations to earlier reports about mega-journals. Hence it was a bit like detective work and there is a risk that some recent journals, which could have fitted the criteria, may have been missed.

\section{Results}

\section{List of megajournals}

The basic information about the fourteen identified journals is shown in table 2 below. There is a clear dominance of biomedicine in terms of topics. Several of the journals already have impact factor and other will get them very soon.

Table 2 The mega-journals studied with basic information about them.

The list of potential journals was longer but journals were dropped due to a number of reasons. One was that some candidate journals don't explicitly have a review process using the soundness only review criterion. Hindawi's Scientific World Journal, which published almost 3,000 articles in 2014, was not included for this reason. ELife fullfils some of the other criterions, but is aiming to be highly selective in prejudging the impact of an article. Also starting journals like Modern languages Open (Liverpool University Press), Open Linguistics (De Gruyter) and Science Advances (AAAS) were not included for the same reason. Brill Open Humanities (Brill) and Heliyon (Elsevier) would fill the criteria but have not started publishing yet. Optics Express has a high publication volume, but a very narrow subject field. Many so-called predatory publishers have journals with extremely broad subject areas aiming at high volumes (Beall, 2013), but they don't fulfill some of the other criteria on the list, in particular since their peer review practices often are highly deficient.

\section{Article volumes}

The year 2010 was picked as the starting point for the collection of article volumes, because this was the year that competitors for PLOS ONE appeared on the market. Two methods were used to determine the yearly article numbers. For journals indexed in Scopus a targeted search for articles and reviews was made in that index. This yielded quantitative results for most of the journals with big publication volumes. For the year 2014 some of the bigger journals were also checked from their web sites, since the Scopus figures for the last year did not appear to be complete at the time of analysis. For the rest of the journals their websites were studied to obtain the article numbers. The figures for 2015 are an estimate based on multiplying the articles published in the first quarter of the year by four. The longitudinal development, is shown in table 3 below. The order is descending according to the total number of articles. It should be noted that at present only six journals have volumes above 500 articles. 
Table 3 Development of article volumes in mega-journals 2010-2015. The figures for 2015 are the articles published in the first quarter of the year multiplied by four.

These statistics seem to indicate that the total output of the mega-journals reached a plateau in 2013 after which PLOS has started to los market share, in particular to Scientific Reports.

\section{Publications charges (APCs)}

The APCs of the journals studied varied in the range 195 - 1950 USD, with several journals situated close to the 1350 USD charged by PLOS ONE. The average was 1300 USD. This level can be compared to a global average for OA journals of around 900 USD (Solomon \& Björk, 2012), charges of 2500-5000 USD in top-ranking OA journals and the general APC level of 3000 USD in hybrid OA journals (Björk, 2012).

Most of the mega-journals have announced a price and kept it unchanged. A notable exception is SAGE Open, which quite dramatically lowered its APC from the initial 695 to 99 USD in 2013. The reason given for this was that an author survey (Research Information, 2013) revealed that most authors who are in the social sciences and humanities had to pay the APC out of their own pocket (currently the APC of Sage Open is 195 USD).

PeerJ offers a membership model, where the author pays a one-time fee and can publish as many articles as they wish. The fee varies according to have many articles per year are allowed, and varies between 99 for one article per year and 299 USD for an unlimited number. Every coauthor needs, however, to be a member. The de facto APC totally paid per article is difficult to calculate because it also depends on the number of repeat authors. Davis (2014) in an analysis of 600 articles published in the journal found a median number of four authors per article, which would indicate an average per article cost of 400 USD. This would be a probably upper limit to the long run average APC.

\section{Acceptance rates}

The only way to obtain information about the acceptance rates for individual journals is if the publisher in question has made this information available. Acceptance rate data could be found for the following journals:
PLOS ONE, $69 \% 3$
BMJ Open, $60 \% 4$
Scientific Reports, $55 \% 5$
FEBS Bio Open, 68\%, 6
Biology Open, $51 \%,{ }^{7}$

\footnotetext{
${ }^{3}$ http://www.plosone.org/static/information

${ }^{4}$ http://bmjopen.bmj.com/site/about/

${ }^{5}$ http://occamstypewriter.org/trading-knowledge/2012/07/09/megajournals/

${ }^{6} \mathrm{http}: / /$ occamstypewriter.org/trading-knowledge/2012/07/09/megajournals/

${ }^{7}$ http://bio.biologists.org/site/about/about_bio.xhtml
} 
These acceptance rates can be compared to the acceptance rates of scholarly journals in general. Although some top journals have acceptance rates of only 5-10 \%, Sugimoto et al (2013) in a study of around 5000 journals in five broad areas found average acceptance rates of between 30 $\%$ (business) and $46 \%$ (health). The corresponding acceptance rates for OA journals in these same fields were between $37 \%$ and $57 \%$.

What this means is that risk of rejection in a mega-journal is significantly lower than in the average peer-review journal in its field. Authors who are reasonably competent as researchers probably perceive the rejection risk as very low, and also much less prone to the subjective values and possible biases of the reviewers asked also to judge the significance of the results in more selective journals.

\section{Publishing speed}

Some mega-journal publishers have made information about acceptance and publishing public on the journal website, in press releases, editorials, conference presentations etc. Scientific Reports for instance announces a median time of 139 days for February 2015 articles ${ }^{8}$. BMJ Open reports a median time to first decision of 46 days in 2013, but there is no info of the full publishing delay ${ }^{9}$. For articles published in December 2014 in PLOS ONE it took 123 days from submission to acceptance and 30 days more to final publication (Davis, 2015). Springer Plus claims a speed to first decision of less than three months and less than a week from acceptance to publication $^{10}$. For articles published in the first half year of PeerJ the median time from submission to final acceptance was 51 days $^{11}$.

Based on the figures above mega-journals seem to have shorter throughput times also compared to OA journals in general, not to mention subscription journals. So-called predatory OA journals seem to be even quicker than mega-journals (Shen \& Björk, 2015), but they often lack a proper peer review and lack academic credibility.

\section{Secondary features}

Table 4 shows the inclusion of the secondary features in the studied journals. In the case of Brill Open Humanities and Helyion it was difficult to check for some of the features since they are not yet publishing articles. It is highly likely that all journals fulfill the publishing speed criterion, but for those were no statistics could be found a "?" has been inserted in the table.

Table 4. Secondary features in use in the studied mega-journals

PeerJ isn't backed by an established publisher, but on the other hand its founders and management team includes individuals with a proven track record in scholarly publishing.

\footnotetext{
${ }^{8} \mathrm{http} / / /$ www.nature.com/content/srep/statistics/index.html?WT.mc_id=WEB_SciReports_2014_LP

${ }^{9}$ http://bmjopen.bmj.com/site/about/

${ }^{10} \mathrm{http}: / /$ cofactorscience.com/blog/journal/springerplus

${ }^{11}$ https://peerj.com/blog/post/60259877854/how-fast-is-peerj/
} 


\section{Discussion}

273 Fourteen mega-journals were identified. In some cases the publishing volumes are still rather

274 small, but given the backing of well-known publishers and the high probability for getting 275 impacts factors in the near future the growth potential is there. The Mega-journal as type of 276 scholarly journals has emerged rapidly, as demonstrated by the growth in publication volumes 277 reported in table 3. Publishers have identified a clear market demand for this type of publication outlet and are offering their services at a price level attracting lots of submissions. A key competitive factor in launching a mega-journal is if the journal can use the publisher's brand to create a high-quality image from the start, hence names like BMJ Open, Springer Plus, SAGE Open. A mega-journal published by a major publisher can also benefit from a ready IT infrastructure for review and manuscript management.

On the other hand the first rapid growth seems to have leveled out and competitors of market leader PLOS ONE seem to be capturing an increasing share. At the current level of around just under 40,000 annual articles the output still only represents some $2 \%$ of the yearly global output of scholarly articles, which is around 2 million (Ware \& Mabe, 2015). The market share of mega-journals of the APC financed Open Access market is however much bigger. Extrapolating from the figure of 136,000 and growth rate reported in Laakso \& Björk (2012) as well as for the figure of 120,000 articles by OASPA member publishers (Redhead ,2014) a conservative estimate for DOAJ registered OA journals would be 200,000 which would mean that the share of megajournals would be in the order of 15-20\%. The future development of mega-journals could be highly dependant on the growth of APC charging OA journals in general.

Mega-journals have found a place in the scholarly publishing ecosystem where they get a fair share of their manuscripts via rejections from other journals higher up in the ecosystem (usually highly selective journals). Solomon (2014) in his survey of authors in four mega-journals found resubmission rates of between 32 and $62 \%$, depending on the journal. The use of portable reviews means that in many such cases the authors lose relatively little time in getting published. One important aspect of mega-journals is how they complement the portfolios of major commercial and society publishers consisting of subscription journals, most of which nowadays offer a hybrid OA choice, and niche and more selective Open Access journals. The mega-journals can use the same technical infrastructures as the other journals, and provide an easy way for the publishers to tap into the growing APC market. The rapid launching of mega-journals of by many established publishers indicates that they are seen as commercially viable. Each major publisher can, however, credibly have only one mega-journal in its portfolio, which poses a limit to the viable number of journals with strong publisher branding.

Although mega-journals offer excellent economies of scale, the growth could in the longer run be limited by the availability of motivated unpaid reviewers (Buriak, 2015). Firstly the task is less intellectually challenging than in more selective traditional journals, where the perceived contribution needs to be judged as well. Secondly the major motivation for unpaid reviewers is often to build up their social capital in their networks of colleagues, so that responding diligently to review requests from leading academics in their field, may lead to appointments to editorial boards, associate editors etc. This factor may be less present in many mega-journals, which rely more on the sense of academic duty of potential reviewers. 
Another aspect limiting the growth is the lack of branding for authors publishing in megajournals. Many experts, promotion committees etc base their evaluations of junior academics primarily on where these have published. In most fields there are well-established pecking orders of top journals, and in addition having a reasonably good impact factor also counts. Academics also tend to spread their articles over a range of journals, where one or even two mega-journal articles can be acceptable, but where numerous ones in the list of publications would be not be good sign.

\section{Acknowledgements}

Tea Mäkelä helped gather publication volume data from journal web sites, and her help is greatly appreciated.

\section{References:}

Beall J. 2013. Five Predatory Mega-Journals: a Review, The Charleston Advisor, 14(4):20-25

Buriak J. 2015. Mega-Journals and Peer Review: Can Quality and Standards Survive? Editorial, Chemistry of Materials 27(7):2243-2243

Binfield P. 2013. Open Access Megajournals - Have They Changed Everything? Blog item, 23.10.2013, Creative Commons, Available at http://creativecommons.org.nz/2013/10/openaccess-megajournals-have-they-changed-everything/ (Accessed 23 April 2015)

Björk B-C. 2012. The hybrid model for open access publication of scholarly articles - a failed experiment? Journal of the American Society for Information Sciences and Technology, 63(8):1496-1504

Björk, B-C, Solomon, DJ. 2013. The publishing delay in scholarly peer-reviewed journals. Journal of Informetrics, 7(4):14-923

Björk B-C, Solomon, DJ. 2014, Developing an Effective Market for Open Access Article Processing Charges, Report, Wellcome Trust, London, United kingdom, 12.3.2014, Available at http://www.wellcome.ac.uk/stellent/groups/corporatesite/@policy_communications/documen ts/web_document/wtp055910.pdf (Accessed 23.4.2015)

Clarke M. (2013) Game of Papers: eLife, BMC, PLoS and EMBO Announce New Peer Review Consortium, 15.7.2013, Available at http://scholarlykitchen.sspnet.org/2013/07/15/game-ofpapers-elife-bmc-plos-and-embo-announce-new-peer-review-consortium/ (Accessed 23 April 2015)

Davis P. (2010) Cascading Peer Review - The Future of Open Access? blog post, Scholarly Kitchen Blog, 12.10.2010, Available at http://scholarlykitchen.sspnet.org/2010/10/12/cascading-peerreview-future-of-open-access/ (Accessed 23 April 2015) 
359 Davis P. (2015) Production Plummets at PLOS-But For a Good Reason, blog post, Scholarly 360 Kitchen Blog, 11.2.2015, Available at

361 http://scholarlykitchen.sspnet.org/2015/02/11/publication-plummets-at-plos-one/ (Accessed

36223 April 2015)

363

364

365

366

367

368

369

370

371

372

373

374

375

376

377

378

379

380

381

382

383

384

385

386

387

388

389

390

391

392

393

394

395

396

397

398

399

Frank (2012) Megajournals, blog post, Occam's typewriter, Available at, http://occamstypewriter.org/trading-knowledge/2012/07/09/megajournals/ (Accessed 23 April 2015)

Laakso M, Björk B-C. 2012. Anatomy of open access publishing - a study of longitudinal development and internal structure, BMC Medicine, 10:124, doi: 10.1186/1741-7015-10-124 Priem J, Taraborelli D, Groth P, Neylon, C. (2010) Altmetrics: a manifesto, 26.10.2010, Available at http://altmetrics.org/manifesto/ (Accessed 23 April 2015)

Redhead C. 2014. Growth of Fully OA Journals Using a CC-BY License, Available at http://oaspa.org/growth-of-fully-oa-journals-using-a-cc-by-license/ (Accessed 23 April 2015)

Research Information (2013) SAGE Open lowers APCs for HSS researchers, 24.1.2013, Available at http://www.researchinformation.info/news/news_story.php?news_id=1089 (Accessed 23 April 2015)

Shen C, Björk B-C. 2015. "Predatory" Open Access - an Overview, working paper, Hanken School of Economics, Helsinki, Finland

Solomon D. 2014. A survey of authors publishing in four megajournals. PeerJ. 2:e365, DOI10.7717/peerj.365

Solomon D, Björk, B-C. 2012. A study of open access journals using article processing charges. Journal of the American Society for Information Science and Technology, 63(8):1485-1495

Sugimoto C, Larivière V, Ni C, Cronin B. (2013). Journal acceptance rates: A cross-disciplinary analysis of variability and relationships with journal measures, Journal of Informetrics, 7(4):897906

Ware M, Mabe M. (2015). The STM Report: An overview of scientific and scholarly journal publishing, STM Association, Available at http://www.stm-

assoc.org/2015_02_20_STM_Report_2015.pdf (Accessed 23 April 2015)

Wikipedia (2015) Mega journal, available at http://en.wikipedia.org/wiki/Mega_journal (Accessed 23 April 2015) 


\section{Table 1 (on next page)}

Table 1

Criteria for a mega-journal used in this study 
2

\begin{tabular}{|l|l|l|l|}
\hline Criteria/mentioned in sources & Wikipedia & Binfield & Frank \\
\hline Primary criteria & & & \\
\hline Big publishing volume or aiming for it & $\sqrt{ }$ & $\sqrt{ }$ & $\sqrt{ }$ \\
\hline Peer review of scientific soundness only & $\sqrt{ }$ & $\sqrt{ }$ & $\sqrt{ }$ \\
\hline Broad subject area & $\sqrt{ }$ & $\sqrt{ }$ & \\
\hline Full open access with APC & $\sqrt{ }$ & $\sqrt{ }$ & $\sqrt{ }$ \\
\hline Secondary criteria & & & \\
\hline Moderate APC & & & $\sqrt{ }$ \\
\hline High prestige publisher & & & \\
\hline Academic editors & $\sqrt{ }$ & $\sqrt{ }$ & $\sqrt{ }$ \\
\hline Reusable graphics \& data & & & \\
\hline Altmetrics & & & $\sqrt{ }$ \\
\hline Commenting & & & $\sqrt{ }$ \\
\hline Portable reviews & & & \\
\hline Rapid publication & $\sqrt{ }$ & & \\
\hline
\end{tabular}

3 


\section{Table 2 (on next page)}

Table 2

The mega-journals studied with basic information about them. 


\begin{tabular}{|l|l|l|l|l|}
\hline Journal & $\begin{array}{l}\text { APC } \\
\text { USD }\end{array}$ & Publisher & Subject field & $\begin{array}{l}\text { Impact } \\
\text { factor }\end{array}$ \\
\hline AIP Advances & 1350 & American Institute of Physics & Physics & 1.6 \\
\hline Biology Open & 1350 & The Company of biologists & Biology & WoS \\
\hline BMJ Open & 1875 & BMJ Publishing Group & Medicine & 2.1 \\
\hline Elementa, Science of the Antropocene & 1450 & BioOne & Earth Sciences & \\
\hline FEBS Open Bio & 1200 & Elsevier & Molecular sciences & WoS \\
\hline G3 & 1950 & Genetics Society of America & Genetics & 2.5 \\
\hline IEEE Access & 1750 & IEEE & Electronics & \\
\hline Journal of Engineering & 1150 & IET & Engineering & \\
\hline PeerJ & 400 & PeerJ & Biology, medicine & WoS \\
\hline PLOS ONE & 1350 & PLOS & Science, medicine & 3.5 \\
\hline Royal Society Open Science & 1600 & Royal Society & All sciences & \\
\hline SAGE Open & 195 & SAGE & Social Science & \\
\hline Scientific Reports & 1495 & Nature Publishing Group & Natural sciences & 5.1 \\
\hline Springer Plus & 1085 & Springer & All sciences & WoS \\
\hline
\end{tabular}




\section{Table 3(on next page)}

Table 3

Development of article volumes in mega-journals 2010-2015. The figures for 2015 are the articles published in the first quarter of the year multiplied by four. 


\begin{tabular}{|c|c|c|c|c|c|c|c|}
\hline \multirow{2}{*}{$\begin{array}{l}\text { Journal/ } \\
\text { articles per year }\end{array}$} & 2010 & 2011 & 2012 & 2013 & 2014 & 2015 & All years \\
\hline & & & & & & & \\
\hline PLOS ONE & 6913 & 13833 & 23441 & 31882 & 30054 & 22120 & 128243 \\
\hline Scientific Reports & & 211 & 800 & 2553 & 3941 & 7692 & 15197 \\
\hline BMJ Open & & 109 & 655 & 959 & 1037 & 1080 & 3840 \\
\hline Springer Plus & & & 82 & 692 & 762 & 612 & 2148 \\
\hline AIP Advances & & 255 & 380 & 396 & 509 & 548 & 2088 \\
\hline PeerJ & & & & 232 & 471 & 636 & 1339 \\
\hline G3 & & 65 & 257 & 250 & 272 & 176 & 1020 \\
\hline SAGE Open & & 44 & 113 & 225 & 217 & 360 & 959 \\
\hline Biology Open & & & 143 & 162 & 137 & 160 & 602 \\
\hline FEBS Open Bio & & 4 & 52 & 78 & 120 & 120 & 374 \\
\hline Royal Society Open Science & & & & & 50 & 196 & 246 \\
\hline IEEE Access & & & & 63 & 106 & 64 & 233 \\
\hline Journal of Engineering & & & & 20 & 102 & 80 & 202 \\
\hline Elementa & & & & 13 & 16 & 28 & 57 \\
\hline All journals & 6913 & 14521 & 25923 & 37525 & 37794 & 33872 & 156548 \\
\hline
\end{tabular}




\section{Table 4(on next page)}

Table 4

Secondary features in use in the studied mega-journals 


\begin{tabular}{|c|c|c|c|c|c|c|c|c|c|}
\hline \multirow{8}{*}{ Journal/ Features } & \multicolumn{9}{|c|}{ Moderate APC } \\
\hline & & \multicolumn{8}{|c|}{ High prestige publisher } \\
\hline & & & \multicolumn{7}{|c|}{ Academic editors } \\
\hline & & & & \multicolumn{6}{|c|}{ Reusable graphics \& data } \\
\hline & & & & & \multicolumn{5}{|c|}{ Altmetrics } \\
\hline & & & & & & \multicolumn{4}{|c|}{ Commenting } \\
\hline & & & & & & & \multicolumn{3}{|c|}{ Portable reviews } \\
\hline & & & & & & & & & publ. \\
\hline AIP Advances & $\sqrt{ }$ & $\sqrt{ }$ & $\sqrt{ }$ & $\sqrt{ }$ & $\sqrt{ }$ & $\sqrt{ }$ & $\sqrt{ }$ & $?$ & \\
\hline Biology Open & $\sqrt{ }$ & $\sqrt{ }$ & $\sqrt{ }$ & $\sqrt{ }$ & $\sqrt{ }$ & & $\sqrt{ }$ & $?$ & \\
\hline BMJ Open & & $\sqrt{ }$ & & $\sqrt{ }$ & $\sqrt{ }$ & $\sqrt{ }$ & & $\sqrt{ }$ & \\
\hline Elementa & $\sqrt{ }$ & $\sqrt{ }$ & $\sqrt{ }$ & $\sqrt{ }$ & $\sqrt{ }$ & & & $?$ & \\
\hline FEBS Open Bio & $\sqrt{ }$ & $\sqrt{ }$ & $\sqrt{ }$ & $\sqrt{ }$ & $\sqrt{ }$ & & & $?$ & \\
\hline G3 & & $\sqrt{ }$ & $\sqrt{ }$ & $\sqrt{ }$ & $\sqrt{ }$ & $\sqrt{ }$ & $\sqrt{ }$ & $?$ & \\
\hline IEEE Access & & $\sqrt{ }$ & $\sqrt{ }$ & & $\sqrt{ }$ & $\sqrt{ }$ & & $?$ & \\
\hline Journal of Engineering & & $\sqrt{ }$ & $\sqrt{ }$ & & $\sqrt{ }$ & $\sqrt{ }$ & & $?$ & \\
\hline PeerJ & $\sqrt{ }$ & & $\sqrt{ }$ & $\sqrt{ }$ & $\sqrt{ }$ & $\sqrt{ }$ & $\sqrt{ }$ & $\sqrt{ }$ & \\
\hline PLOS ONE & $\sqrt{ }$ & $\sqrt{ }$ & $\sqrt{ }$ & $\sqrt{ }$ & $\sqrt{ }$ & $\sqrt{ }$ & $\sqrt{ }$ & $\sqrt{ }$ & \\
\hline Royal Society Open Science & & $\sqrt{ }$ & $\sqrt{ }$ & $\sqrt{ }$ & $\sqrt{ }$ & & $\sqrt{ }$ & $?$ & \\
\hline SAGE Open & $\sqrt{ }$ & $\sqrt{ }$ & & $\sqrt{ }$ & $\sqrt{ }$ & $\sqrt{ }$ & $\sqrt{ }$ & $?$ & \\
\hline Scientific Reports & $\sqrt{ }$ & $\sqrt{ }$ & $\sqrt{ }$ & $\sqrt{ }$ & $\sqrt{ }$ & & $\sqrt{ }$ & $\sqrt{ }$ & \\
\hline Springer Plus & $\sqrt{ }$ & $\sqrt{ }$ & & $\sqrt{ }$ & $\sqrt{ }$ & $\sqrt{ }$ & $\sqrt{ }$ & $\sqrt{ }$ & \\
\hline
\end{tabular}

